lights so that the illumination level can be varied. Under those conditions, a cardboard backing for each stimulus would be needed to prevent external light, which penetrates the card, from changing the inside illumination.

Since $3 \times 5$ index cards are used, it is a simple matter to make stimuli; the distance from the shutter to the card is 11 in. making typewriter letters readily visible. The particular shutter suggested can vary duration of exposure from $1 \mathrm{sec}$ through $1 / 100 \mathrm{sec}$; the opening of the diaphragm can be varied as well. The two primary disadvantages of this apparatus are that no focal point is provided other than the center of the diaphragm itself, and that the S's eye tends to dark-adapt if brought too close to the shutter.

The shutters mentioned above have been used for a number of years without any appreciable wear. The apparatus is superior to those using a "guillotine" door (Bugelski, 1951), the sectored rotating disk (Ferree \& Rand, 1937), and the dual mirror arrangement (Lvans, 1931) because of its simple, compact desigr and because both duration of exposure and intensity $o$ illumination can be varied systematically. Users must, however take note that leaf shutters such as this are not reliable enougl for research, since exposures at a given setting vary by as much a $10 \%$.

\section{REFERENCES}

BEHR, I. A new tachistoscope for animals and man. American Journal o Psychology, 1960, 73, 305-306.

BUGELSKI, B. R. Experimental psychology. New York: Holt \& Company 1951. Pp. 83-84.

EVANS, J. E. A tachistoscope for exposing large areas. American Journa of Psychology, 1931, 43, 285-286.

FERREE, C. E., \& RAND, G. A multipleexposure tachistoscope. Journa of Experimental Psychology, 1937, 21, 240-259.

HOWARD, I. P., \& EVANS, J. A. A new splitbeam tachistoscope giving a exact color-match. American Journal of Psychology, 1964, 77, 311-312

\title{
A device for rapid recording of positioning responses in two dimensions'
}

JOSEPH A. BAUER, JR., GORDON D. WOODS, and RICHARD HELD, DEPARTMENT OF PSYCHOLOGY, MASSACHUSETTS INSTITUTE OF TECHNOLOGY, Cambridge, Massachusetts 02139

A device for rapid recording of positioning responses is described. It utilizes an extremely simple, low-cost position transducer and two digital voltmeters or their equivalent. The device has the advantages of rapid, accurate measurement, reliability, ease of reading, and the potential for automatic recording of positional data in two dimensions.

In the study of sensorimotor coordination, pointing at a target is often used as a response and the appropriate response measure is the position of the tip of the index finger relative to the target position or to some arbitrary origin (Efstathiou, Bauer, Greene, \& Held, 1967; Holding, 1968; Keele \& Posner, 1968). If the $S$ holds a pencil in his hand while pointing, the position at which he touches it to a surface can be recorded on a piece of paper. However, the use of pencil marks on paper has three major disadvantages: First, as the number of data points increases, the problem of distinguishing overlapping markings arises. Secondly, the sequential order of markings is lost unless each response is numbered as it is made. Thirdly, as the amount of data collected increases, the time required for numerical transcription increases rapidly even if positions of marks are measured in only one direction (i.e., horizontal). It takes far longer for the $\mathrm{E}$ to transcribe a response than for the $S$ to make it. For these reasons, we have designed and constructed a system for rapid numerical recording in two dimensions of the end position of a response.

The system consists of three interconnected functional units. The first is the position transducer, which provides an electrical signal proportional to position. The second is a voltmeter, which measures and displays the signal from the transducer. The third is a control unit, which governs the sequential operation of the other two. Except for the position transducer, the actual choice of components is determined by the degree of automation desired for data acquisition and the estimated total cost of the system.

A scheme for implementing Teledeltos ${ }^{2}$ paper in a two-dimensional position transducer has been developed by
Blount (1967). Teledeltos paper is a metalized sheet of unifom electrical resistance in all directions across its surface. It can $b$ bonded to plywood or hardboard with photographic $\mathrm{dr}$. mounting tissue. Figure 1 shows electrical connection of Teledeltos paper-covered board. The appropriate leads of th diodes are inserted through holes in the board spaced at 1 -in intervals. A spot of conducting paint at each junction make electrical connection between the diode and paper. When the bia voltage (V) is positive, as defined in Fig. 1, current will flow onl in the horizontal direction through the diodes along the vertic: sides. The current flow is uniform across vertical sections of th surface because of the spacing of the diodes, and the diodes alon

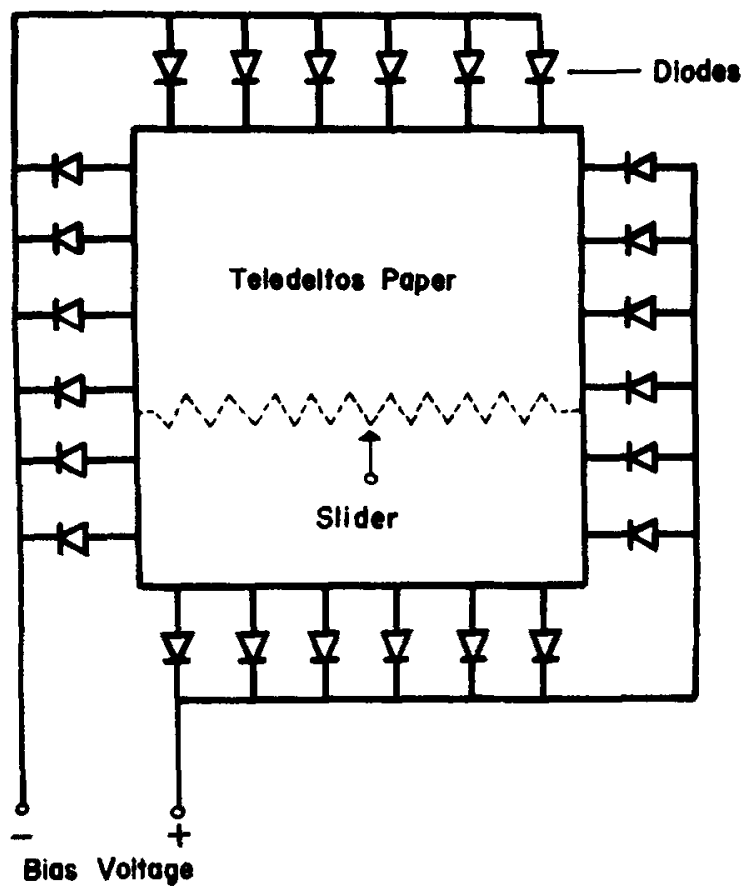

Fig. 1. Teledeltos board connections. 


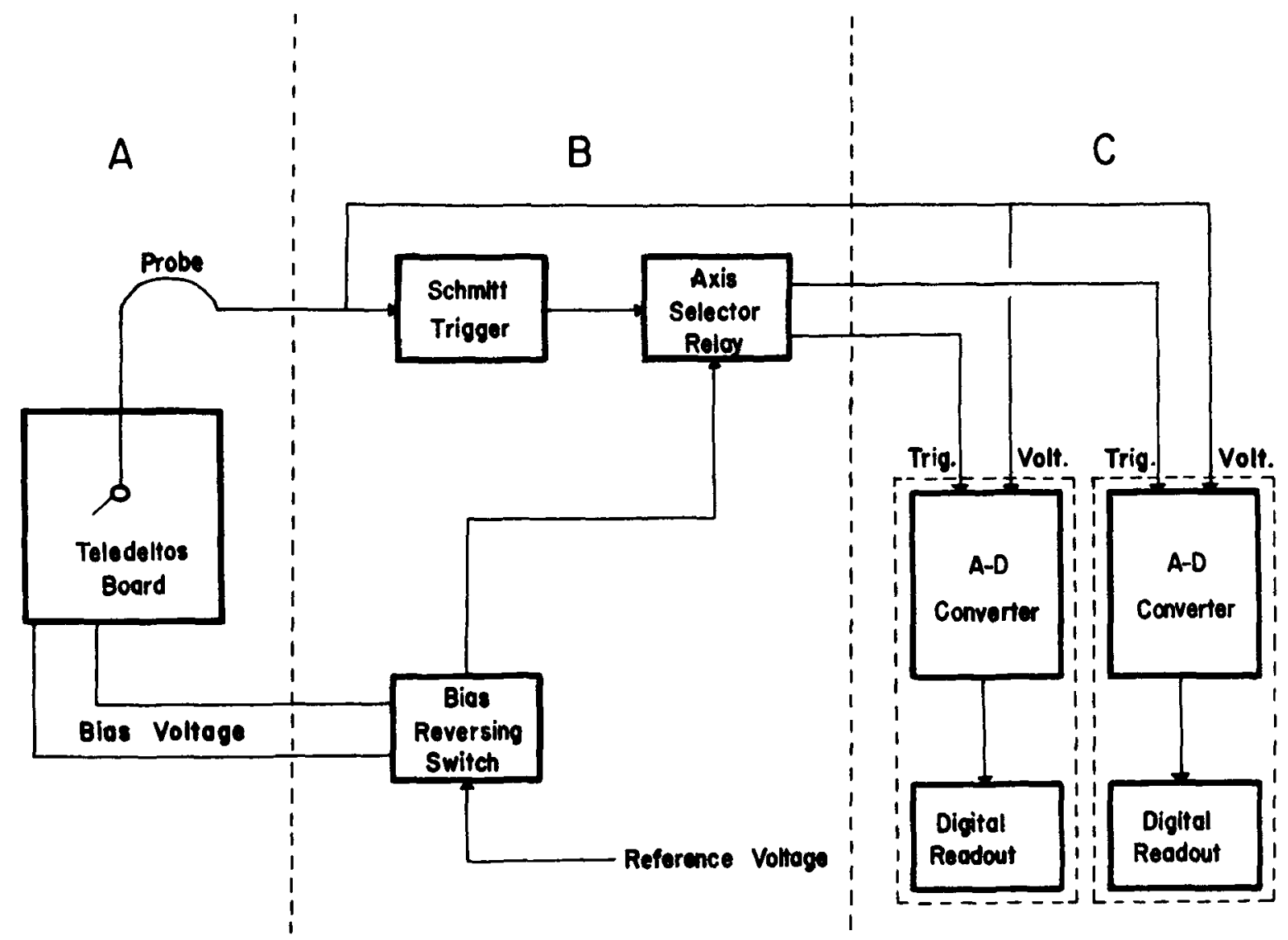

Fig. 2a. Dual DVM system.

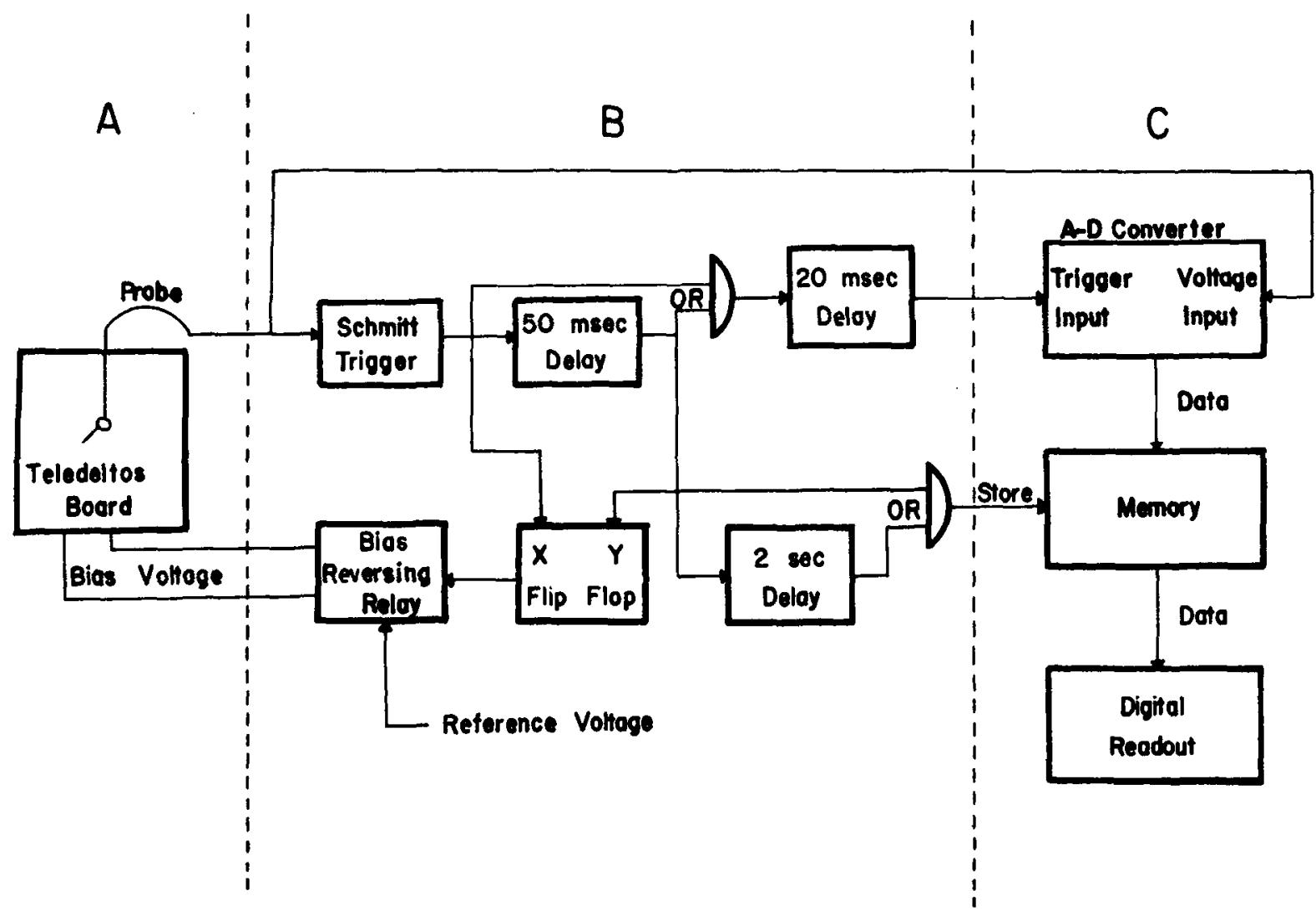

Fig. 2b. Single converter system. Section A is the transducer; B, the control unit; and C, the A-D converter and digital readout. 
the horizontal sides are reverse biased so that no current flows through them. The current passing through the resistance paper produces a voltage drop that increases linearly across the paper. By reversing the polarity of the bias voltage applied to the board, a vertical current is produced with a resulting vertically increasing voltage drop. The voltages, which are proportional to position, are then measured by a circuit completed by contact of a conducting probe held by the S. In effect, each axis of the Teledeltos paper may be modeled as a potentiometer with the probe as the slider arm, as shown by the dotted line in Fig. 1 .

\section{Voltmeter}

Several devices could be used to measure the signal generated by the position transducer. Standard Teledeltos paper has a thin insulating coating that must be pierced by a pointed probe to obtain a reliable measurement. ${ }^{3}$ If a voltmeter is used, it must have a high-input resistance to minimize errors resulting from differences in pressure applied to the probe by the S. A standard moving-coil voltmeter could be used but is undesirable since it generally does not have the required accuracy, is tedious to read repeatedly, and requires a skilled operator. The use of an analog-to-digital (A-D) converter and a numerical readout device overcemes these difficulties because once the voltage is measured, it remains displayed after the input has been disconnected. A digital voltmeter (DVM) combines both devices in a single compact and readily available unit. In addition, a digital device has sufficient accuracy so that adjustment of the bias voltage can yield readings that are in fractions of an inch or centimeter. For example, a Teledeltos board, $20 \times 20$ in., could be supplied by a $20-\mathrm{V}$ bias voltage. A three-digit DVM set on the $100-\mathrm{V}$ range would then give readings that would correspond to position to the nearest tenth of an inch without conversion.

\section{Control Unit}

The design and construction of the control unit depend on the number and kind of voltage measuring and display devices utilized in the system. With a single DVM the most straightforward control unit would consist of a probe-activated trigger to the DVM and a manually operated bias reversing switch that would also retrigger the DVM for a second reading. This method requires the $E$ to record the first reading before triggering the second. Consequently, $S$ must maintain contact with the board until the second reading is completed. If two DVMs were used, they could be triggered in rapid succession even with a manually operated bias reversing switch and $S$ would not have to maintain prolonged contact with the board during recording of the data. A block diagram of such a system is shown in Fig. 2a. The dotted-line boxes in Section C each represent a DVM. The substitution of an automatic control unit for the bias switch would speed up the reading even further. This system can be readily implemented with commercially available components, but two DVMs are a considerable luxury.

The system shown in Fig. $2 \mathrm{~b}$ has an automatic control unit and uses one A-D converter, one digital readout, and an in tegrated circuit memory instead of two DVMs. Consequently, the control unit is more complex than that for the dual DVM system. In this system, the probe activates the control unit that triggers the first A-D conversion and transfers it to the memory where it is stored and displayed. Then it reverses the bias voltage, makes another A-D conversion for the second axis, and stores this reading in the converter. All this can be accomplished in $100 \mathrm{msec}$ or less. After the first valuc has been recorded, the contents of the converter are transferred to the memory where they are displayed until the probe contact activates the control unit again. In this case, the first value is displayed for a fixed interval of $2 \mathrm{sec}$-ample time for a three-digit number to be read for recording by pencil and paper. The main advantage of automatic control is that other control functions, such as automatic selection and presentation of targets, can be added easily to the existing control unit.

Although the control unit in Fig. $2 b$ seems to be the most complex part of the system, the major cost of either system lies in the A-D converter. The advantage of the first system is its simple design using readily available, though expensive, components. The second system uses only one A-D converter at the expense of a more complicated control unit and a memory. However, the present cost and availability of integrated circuit logic components makes the total cost and complexity of the second system lower than that of the first.

A system using the single converter method has been developed using integrated circuit logic and a hybrid vacuum tube-transistor A-D converter. The hybrid A-D device is a voltage-to-pulse width converter that generates a gating pulse whose duration varies linearly with the input voltage. The digital conversion of the input voltage is accomplished by counting the number of pulses from a $100 \mathrm{KC}$ crystal oscillator that occur during the time width of the gating pulse. The count measured is then displayed by numerical indicator lights. The system will measure to within $\pm 1 / 10$ in. on a $30 \times 30$-in. surface. The integrated circuit control unit performs all the operations described above for the single A-D converter system with two important additions. First, it selects and presents target lights on a preprogrammed basis. Secondly, it controls the operation of an IBM-024 card punch during the experimental session. After each pointing response of the $S$ to the presentation of a target, the target number and a three-digit number of each of the $X$ and $Y$ coordinates of the response are punched onto an IBM card.

A modified version of this system without the target selector and card punch control functions has been in operation in our laboratory for over a year. During this period it has been used in a variety of experiments by people of widely differing degrees of electronic sophistication. All of them have been impressed by its reliability. Periodic calibration checks have revealed that its accuracy over the same period has been remarkably stable. Thus, for anyone desiring to record the positional attributes of a reaching or aiming response in either one or two dimensions, the device described above is eminently suitable in terms of both cost and performance.

\section{REFERENCES}

BLOUNT, F. I. Design of a low cost computer graphical input device. Unpublished undergraduate thesis. MIT Engineering Library, 1967.

EFSTATHIOU, A., BAUER, J., GREENE, M., \& HELD, R. Altered reaching following adaptation to optical displacement of the hand. Journal of Experimental Psychology, 1967, 73, 113-120.

HOLDING, D. H. Accuracy of delayed aiming responses. Psychonomic Science, 1968, 12, 125-126.

KLELE, S. W., \& POSNER, M. I. Processing of visual feedback in rapid movement. Joumal of Experimental Psychology, 1968, 77, 155-158.

\section{NOTES}

1. The development of this device was supported by NIMH Grant MH-07642 and NASA Grant NsG496.

2. Teledeltos paper may be obtained from Western Union Telegraph Company, 60 Hudson Street, New York, New York 10013.

3. Type L-black Teledeltos paper does not have the thin insulating coating and need only be contacted with a rounded metallic probe, such as an empty ball point pen, for reliable measurement. 